\title{
Association between C-Reactive Protein Rapid Test and Group A Streptococcus Infection in Acute Pharyngitis
}

\author{
Olga Calviño, MD, Carl Llor, PhD, Frederic Gómez, PhD, Eva González, MD, \\ Carolina Sarvisé, MD, and Silvia Hernández, PhD
}

Introduction: The diagnosis of streptococcal infection is usually made with the use of Centor criteria, but some family doctors also rely on the determination of C-reactive protein (CRP) to guide antibiotic therapy.

Methods: This was an observational study conducted in a health center. Adults with acute pharyngitis and the presence of the 4 Centor criteria (tonsillar exudates, tender cervical glands, history of fever, and absence of cough) were recruited. The patients underwent a pharyngotonsillar swab for microbiologic study and a CRP rapid test during the consultation.

Results: A total of 149 patients were enrolled. The most frequent etiology was group A streptococcus, present in 83 cases (55.7\%). The highest CRP concentration was observed among patients with group $\mathrm{C}$ streptococcus infection, with a mean of $56.3 \mathrm{mg} / \mathrm{L}$ ( $95 \%$ confidence interval, $25.7-86.5 \mathrm{mg} / \mathrm{L})$. For patients with group A streptococcus infection, the mean CRP value was 34.4 (95\% confidence interval, $25.6-43.3 \mathrm{mg} / \mathrm{L})$.

Conclusion: CRP concentrations are not associated with group A streptococcus infection in patients with acute pharyngitis. The use of this point of care test is therefore not useful for distinguishing patients who require antibiotic therapy. (J Am Board Fam Med 2014;27:424-426.)

Keywords: Antibiotics, C-Reactive Protein, Infectious Diseases, Pharyngitis, Streptococcus Group A

Point-of -care testing for C-reactive protein (CRP) was introduced into community clinical practice in the early 1990s and is widely used in Scandinavian countries. ${ }^{1}$ This test provides results in about 3 minutes and guides decisions regarding antibiotic treatment. Several randomized clinical trials and

This article was externally peer reviewed.

Submitted 10 December 2013; revised 9 February 2014; accepted 11 February 2014.

From the Institute of Primary Care and Public Health, Cardiff University School of Medicine, Cardiff, UK (CL); Primary Care Center Jaume I, Tarragona, Spain (OC, SH); and the Department of Laboratory and Microbiology, Hospital Joan XXIII, Tarragona, Spain (FG, EG, CS).

Funding: none.

Conflict of interest: CL reports having a grant from the Fundació Jordi Gol i Gurina for a research stage at the University of Cardiff. He also reports receiving research grants from the European Commission (Sixth and Seventh Programme Frameworks), Catalan Society of Family Medicine, and Instituto de Salud Carlos III (Spanish Ministry of Health). The other authors have nothing to declare.

Corresponding author: Carl Llor, PhD, Institute of Primary Care and Public Health, Cardiff University School of Medicine, 5th Floor, Neuadd Meirionnydd Heath Park, Cardiff CF14 4XN, UK (E-mail: LlorC@cardiff.ac.uk). observational studies have found that CRP rapid testing significantly reduces the prescription of antibiotics to patients with lower respiratory tract infections ${ }^{2}$; several studies have shown that elevated CRP concentrations are associated with bacterial etiology. ${ }^{3}$ Among patients with acute pharyngitis, only those infections caused by group A streptococcus (GAS) should be treated with antibiotics. ${ }^{4}$ However, some series have shown a high prevalence of non-GAS infection, and complications after infections caused by groups $\mathrm{C}$ and $\mathrm{G}$ streptococci have been reported. ${ }^{5}$ In some countries, this diagnosis is conducted with the aid of rapid antigen detection tests, but because of the uncertainty of infections caused by streptococci other than GAS, some physicians also rely on CRP to guide antibiotic treatment, despite the use of this test not being recommended in such situations. ${ }^{1}$ We conducted a study aimed at understanding the relationship between the etiology of sore throat and CRP concentrations in patients with suspected streptococcal infection. 
Table 1. C-Reactive Protein (CRP) Concentrations Based on the Bacteria Causing Pharyngitis in Patients with 4 Centor Criteria

\begin{tabular}{lcrr}
\hline & & \multicolumn{2}{c}{ CRP Concentrations (mg/L) } \\
\cline { 3 - 4 } Organism & Patients, n (\%) & Mean (95\% CI) & Median (IQR) \\
\hline GAS & $83(56.1)$ & $34.4(25.6-43.3)$ & $12.0(4.0-62.0)$ \\
Non-GAS infection* & $65(43.9)$ & $29.9(19.7-40.2)$ & $8.0(4.0-46.0)$ \\
No bacteria & $29(19.6 \%)$ & $27.9(11.0-44.9)$ & $4.0(4.0-37.5)$ \\
Group B streptococcus & $8(5.4 \%)$ & $19.1(0-41.0)$ & $4.0(4.0-36.7)$ \\
Group C streptococcus & $13(8.8)$ & $56.3(25.7-86.9)$ & $61.0(4.0-94.5)$ \\
Group G streptococcus & $5(3.4)$ & $31.6(0-65.3)$ & $38.0(4.0-56.0)$ \\
Other streptococci & $10(6.7)$ & $9.2(4.4-14.0)$ & $8.0(4.0-11.2)$ \\
\hline
\end{tabular}

CI, confidence interval; GAS, group A streptococcus; IQR, interquartile range.

${ }^{*}$ One patient had infection from a bacteria not included here.

\section{Methods}

An observational study was undertaken in an urban health center from January 2010 to May 2012. Adults $\geq 18$ years old with acute pharyngitis and the presence of the 4 Centor criteria ${ }^{6}$ - history of fever, presence of tonsillar exudates or hypertrophy, presence of tender cervical glands, and absence of cough-were consecutively recruited. All patients underwent a pharyngotonsillar swab for microbiologic culture, which was sent to the Department of Microbiology, Joan XXIII University Hospital (Tarragona, Spain) in Amies medium (Copan Innovation, Brescia, Italy). Samples were seeded on a plate of blood agar and were incubated at $37^{\circ} \mathrm{C}$ in an $5 \%$ carbon dioxide atmosphere for $48 \mathrm{~h}$. A culture was considered positive for GAS with a growth of any number of $\beta$-hemolytic colonies, gram-positive staining with streptococcal morphology, and a catalase negative test with posterior identification with an automated panel for grampositive cocci using the Wider System (F. Soria Melguizo, Madrid, Spain). Results were confirmed with posterior serogrouping with the Streptococcal Grouping Kit (Thermo Scientific, Oxford, UK). The culture was considered negative with the absence of $\beta$-hemolytic colonies after 48 hours of incubation. Patients underwent a CRP rapid test during the consultation by means of QuikRead/Go devices (Orion Diagnostica, Espoo, Finland).

\section{Results}

A total of 149 patients were enrolled. The most frequent etiology was GAS, present in 83 cases (55.7\%). No bacteria were identified in 29 patients $(19.5 \%)$. The age of the patients ranged from 18 to
51 years, with a mean of 28.5 years. GAS infection presented a higher CRP concentration compared with other etiologies (34.4 vs $29.9 \mathrm{mg} / \mathrm{L}$, respectively), with no statistically significant differences (Table 1). The highest CRP concentration was observed among patients with group $\mathrm{C}$ streptococcus infection. Infection with groups $C$ and $G \beta$-hemolytic streptococci was associated with slightly higher concentrations of CRP (49.9 mg/L) compared with those with infection by GAS, other causes, or no infection (Figure 1). The highest CRP concentration $(182 \mathrm{mg} / \mathrm{L})$ was observed in a patient presenting with a peritonsillar abscess and no bacterium identified during the consultation.

\section{Discussion}

This study has several limitations. Only patients with the 4 Centor criteria were recruited, which explains why more than half of the patients actually had a GAS infection. Outcomes were not collected, nor was the evolution of symptoms measured, but we do not consider these limitations to be important since our goal was to identify the association of CRP concentrations with the etiology of the pharyngitis. The microbiologic study did not take into account the study of anaerobes, and some of the patients in whom no bacteria were identified may have been infected by these organisms. In our study the CRP rapid test used a finger prick blood sample; however, studies comparing these rapid tests with the routine CRP laboratory test have shown a very good correlation, thereby demonstrating its reliability.

Some studies have shown high CRP concentrations with GAS infection. Hjortdahl and Melbye ${ }^{7}$ observed a mean CRP concentration of $50.4 \mathrm{mg} / \mathrm{L}$ 
Figure 1. Association between C-reactive protein concentrations and the cause of infection among patients with sore throat and 4 Centor criteria.

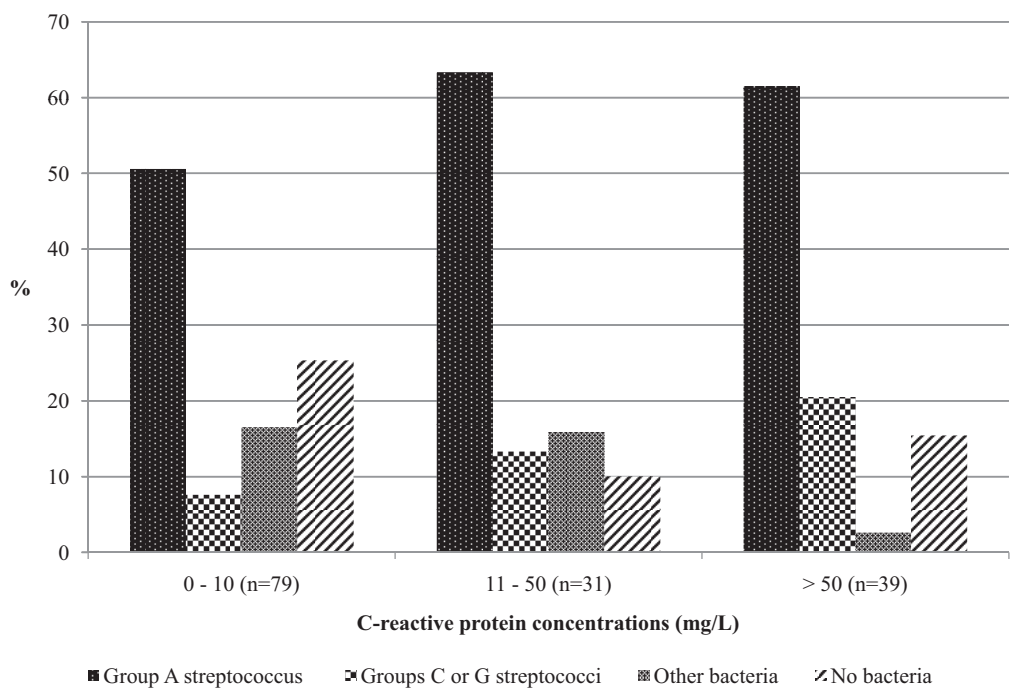

among patients with GAS infection, and Melbye et $\mathrm{al}^{8}$ observed an even higher mean value of 100.3 $\mathrm{mg} / \mathrm{L}$; however, they recruited only 11 patients with GAS. On the other hand, other studies have shown no relationship between CRP concentrations and GAS infection. In a Swedish study, the mean CRP value was $37.6 \mathrm{mg} / \mathrm{L}$ for the group with rapid antigen detection tests showing GAS and $37.4 \mathrm{mg} / \mathrm{L}$ for those with a test not showing GAS. ${ }^{9}$ Lindbæk et $\mathrm{al}^{10}$ observed mean CRP values of 43 $\mathrm{mg} / \mathrm{L}$, with the highest concentrations being found in infections caused by groups C and G streptococci, which is similar to our results.

\section{Conclusion}

Some studies suggest that CRP testing is overused. ${ }^{1,9}$ Our results show that CRP is not useful for differentiating GAS infection from other etiologies that do not require antibiotic therapy.

\section{References}

1. André M, Vernby A, Odenholt I, et al. Diagnosisprescribing surveys in 2000, 2002 and 2005 in Swedish general practice: consultations, diagnosis, diagnostics and treatment choices. Scand J Infect Dis 2008;40:648-54.

2. Huang Y, Chen R, Wu T, Wei X, Guo A. Association between point-of-care CRP testing and antibiotic prescribing in respiratory tract infections: a systematic review and meta-analysis of primary care studies. Br J Gen Pract 2013;63:e787-94.
3. Cals J, van Weert H. Point-of-care tests in general practice: hope or hype? Eur J Gen Pract 2013;19: 251-6.

4. Shulman ST, Bisno AL, Clegg HW, et al; Infectious Diseases Society of America. Clinical practice guideline for the diagnosis and management of group A streptococcal pharyngitis: 2012 update by the Infectious Diseases Society of America. Clin Infect Dis 2012;55:e86-102.

5. Tiemstra J, Miranda RLF. Role of non-group A streptococci in acute pharyngitis. J Am Board Fam Med 2009;22:663-9.

6. Centor RM, Witherspoon JM, Dalton HP, Brody CE, Link K. The diagnosis of strep throat in adults in the emergency room. Med Decis Making 1981;1: 239-46.

7. Hjortdahl P, Melbye H. Does near-to-patient testing contribute to the diagnosis of streptococcal pharyngitis in adults? Scand J Prim Health Care 1994;12: $70-6$.

8. Melbye H, Bjørkheim MK, Leinan T. Daily reduction in C-reactive protein values, symptoms, signs and temperature in group-A streptococcal pharyngitis treated with antibiotics. Scand J Clin Lab Invest 2002;62:521-5.

9. Engström S, Mölstad S, Lindström K, Nilsson G, Borgquist L. Excessive use of rapid tests in respiratory tract infections in Swedish primary health care. Scand J Infect Dis 2004;36:213-8.

10. Lindbæk M, Høiby EA, Lermark G, Steinsholt IM, Hjortdahl P. Clinical symptoms and signs in sore throat patients with large colony variant beta-haemolytic streptococci groups $\mathrm{C}$ or $\mathrm{G}$ versus group $\mathrm{A}$. Br J Gen Pract 2005;55:615-9. 